\title{
Ability of sodium copper chlorophyllin complex to repair photoaged skin by stimulation of biomarkers in human extracellular matrix
}

This article was published in the following Dove Press journal:

Clinical, Cosmetic and Investigational Dermatology

25 July 2016

Number of times this article has been viewed

\author{
John P McCook' \\ Thomas J Stephens ${ }^{2}$ \\ Lily I Jiang ${ }^{2}$ \\ Robert M Law ${ }^{3}$ \\ Vincent Gotz ${ }^{4}$ \\ 'Discovery Partners LLC, Frisco, \\ ${ }^{2}$ Thomas J. Stephens \& Associates, \\ Inc., Richardson, ${ }^{3}$ ProPath, Dallas, \\ TX, ${ }^{4}$ MDRejuvena, Inc., San Diego, \\ CA, USA
}

Correspondence: John P McCook Discovery Partners, LLC, 4913 Southern Hills Drive, Frisco, TX 75034, USA $\mathrm{Tel}+\mathrm{I} 2144486513$

Email johnmccook@att.net
Purpose: To examine the effect of sodium copper chlorophyllin complex on the expression of biomarkers of photoaged dermal extracellular matrix indicative of skin repair.

Patients and methods: Following a previously published 12-day clinical assessment model, skin biopsy samples from the forearms of four healthy females with signs of photoaged skin were obtained and samples were analyzed by immunohistochemistry for key biomarkers of aging skin after each subject was treated with a test material consisting of a gel containing a liposomal dispersion of sodium copper chlorophyllin complex $0.05 \%$, a positive control of tretinoin cream $0.025 \%$, and an untreated negative control.

Results: There was a statistically significantly greater amount of fibrillin/amyloid $\mathrm{P}$ and epidermal mucins found for skin treated with the test material containing $0.05 \%$ sodium copper chlorophyllin complex and the reference control tretinoin $0.025 \%$ cream compared to the negative control (untreated site). Expression of procollagen 1 and dermal mucin also showed a greater presence in the samples treated with the test material and the reference control compared to the negative control, though the differences were not statistically significant. No adverse events were observed or reported by the subjects during the course of the study.

Conclusion: The results of this human biopsy study suggest that both retinoids and sodium copper chlorophyllin complex have beneficial effects on biomarkers of photoaged skin. Products containing both sodium copper chlorophyllin complex and retinols may provide a dual approach to reversing age-related decreases in hyaluronic acid (HA) in the skin: inhibition of the breakdown of HA via sodium copper chlorophyllin complex by inhibition of hyaluronidase, and stimulation of HA synthases by retinol.

Keywords: hyaluronic acid, glycosaminoglycans, retinoid, hyaluronidase, mucins

\section{Introduction}

The extracellular matrix (ECM) is composed of two main classes of macromolecules: proteoglycans, which includes glycosaminoglycan (GAG) chains, primarily hyaluronic acid (HA; hyaluronan); and fibrous proteins, which include collagens, elastins, fibronectins, and laminins. HA is the predominant GAG of human skin, constituting more than $50 \%$ of the total HA in the body. ${ }^{1}$ Because of its negative charge, HA is highly hydrating and serves as an extracellular reservoir that holds large amounts of cations and water, a mechanism for maintaining normal hydration of the skin. With advancing age, HA polymers become more tissue associated, presumably through HA-binding proteins such as fibrinogen, collagen, CD44, and hyaluronidase, which may underlie some of the changes in human skin that occur with aging. ${ }^{2}$ The normal process of 
aging, as well as premature aging caused by sun damage, involve similar mechanisms that cause loss of moisture and changes in HA distribution. ${ }^{3}$ Degradation of HA in the ECM by hyaluronidases results in a breakdown of structural integrity and an increase in tissue permeability, ${ }^{4}$ thus inhibition of hyaluronidase activity prevents rapid degradation of HA and helps in maintaining structural integrity. As agents that maintain HA homeostasis, hyaluronidase inhibitors may serve as antiaging, anti-inflammatory, and antimicrobial agents. ${ }^{5}$

Sodium copper chlorophyllin complex is a semisynthetic mixture of the water-soluble sodium salt of copper chlorophyllin and small molecule analogs derived from plant chlorophyll that possesses a unique combination of anti-inflammatory, antibacterial, and antioxidant activities. ${ }^{6}$ Sodium copper chlorophyllin complex has been used topically for many years to aid in wound healing ${ }^{7-9}$ but the ingredient has not been used until recently as an antiaging cosmeceutical in topical products. Sodium copper chlorophyllin complex has demonstrated inhibition of hyaluronidase activity in vitro, supporting the concept that topical application of sodium copper chlorophyllin complex may be useful in the regulation of HA in the skin, thereby acting to enhance the improvement in the appearance of facial skin. ${ }^{10}$

Topical formulations containing a liposomal dispersion of sodium copper chlorophyllin complex have shown promise in the clinical treatment of various dermatologic conditions. A pilot study demonstrated that sodium copper chlorophyllin complex was clinically effective and well tolerated for the treatment of mild-to-moderate photodamage and solar lentigines when used for 8 weeks. ${ }^{11}$ Another study demonstrated that topical sodium copper chlorophyllin complex was clinically effective and well tolerated for the treatment of mild-to-moderate acne and large, visible pores when used for 3 weeks. ${ }^{12}$ In uncontrolled case studies, topical application of formulations containing sodium copper chlorophyllin complex demonstrated a reduction in facial redness, decreased pore size, and improved skin texture. ${ }^{13}$

The present study was conducted to examine the effect of sodium copper chlorophyllin complex on the expression of biomarkers of photoaged dermal ECM indicative of skin repair. In this study, skin biopsy samples from the forearms of four healthy females with signs of photoaged skin were obtained and samples were analyzed for key biomarkers of aging skin after each subject was treated with a test material consisting of a treatment gel containing a liposomal dispersion of sodium copper chlorophyllin complex, $0.05 \%$ (Phytochromatic MD ${ }^{\circledR}$ Complex; MDRejuvena, Inc., San Diego, CA, USA), a positive control of tretinoin cream,
$0.025 \%$, and a no treatment control. Tretinoin (retinoic acid) can repair photoaged dermal matrix and is regarded as the "gold standard" against which repair agents are judged. ${ }^{14}$

\section{Materials and methods \\ Subjects}

Females aged between 45 and 55 years were eligible to be screened for the study. Prospective subjects completed an eligibility and health questionnaire and were evaluated for eligibility criteria. To qualify for enrollment, subjects were required to have Fitzpatrick skin type II or III, with clinically determined photoaged skin on the extensor surfaces of the forearms.

\section{Study design}

This was a single-center, randomized, controlled clinical trial (clinical trial \#C15-D145) conducted over the course of 12 days to evaluate the ability of a topical formula containing $0.05 \%$ sodium copper chlorophyllin complex to repair photoaged dermal matrix by monitoring changes in reported biomarkers from skin biopsy specimens taken from the outer forearms of subjects. Prior to study enrollment, the protocol, informed consent form, and recruitment materials were approved by the IntegReview Institutional Review Board. Testing was conducted between August 15, 2015, and September 2, 2015, by Thomas J Stephens \& Associates, Inc., Richardson, TX, USA; Stephens Study Number C15-D145.

\section{Test material}

The test material was gel formula DR198-02 containing an aqueous liposomal dispersion of $0.05 \%$ sodium copper chlorophyllin complex.

The cosmetic ingredient declaration to qualitatively describe the gel formula DR198-02 is as follows:

Water (aqua)
Pentylene glycol
Sodium lactate
Butylene glycol
Carbomer
Chlorophyllin-copper complex
Phosphatidylcholine
Phenoxyethanol
Glycerin
Camellia sinensis (green tea) leaf extract
Sodium hydroxide
Sodium ascorbate* (*antioxidant preservative present
at $<1$ ppm)

Water (aqua)

Pentylene glycol

Sodium lactate

Butylene glyco

Carbomer

Chlorophyllin-copper complex

Phosphatidylcholine

Glycerin

Camellia sinensis (green tea) leaf extract

Sodium hydroxide

at $<1 \mathrm{ppm}$ ) 


\section{Study procedures}

The study design used for this trial was modeled after Watson et al, ${ }^{14}$ in which retinoic acid was used as a positive control against which to test the activity of a variety of antiaging products to repair photoaged skin as assessed by the expression of key biomarkers. Qualified subjects had three $4 \times 5 \mathrm{~cm}$ patch sites marked on the extensor surface of the left or right forearm according to a predetermined randomization. On days $1,4,6,8$, and $10,50 \mu \mathrm{L}$ of the test material (formula DR $198-02$ containing $0.05 \%$ sodium copper chlorophyllin complex) was dosed directly onto one of the randomized marked patch sites, for 12 days of exposure. On day $8,50 \mu \mathrm{L}$ of the reference control material (tretinoin $0.025 \%$ cream) was dosed directly onto an assigned patch site, for 4 days of exposure only (to minimize the potential for skin irritation) as described by Watson et al. ${ }^{14}$ The remaining site was untreated during the course of the study to serve as a negative control. All three test sites were covered with Finn Chambers (Allerderm Laboratories, Inc., Mill Valley, CA, USA) $12 \mathrm{~mm}$ inner diameter aluminum chambers affixed to Scanpore Tape (Norgesplaster A/S, Kristiansand, Norway) on each application day.

Clinical evaluations were conducted at visit 2 (day 4), visit 3 (day 6), visit 4 (day 8), visit 5 (day 10), and visit 6 (day 12) for reaction grading and scoring of test sites.

\section{Biopsy procedures and scoring}

Selected subjects had a $3 \mathrm{~mm}$ punch biopsy collected from each of the three test sites at day 12. Biopsies were transferred immediately after collection into $10 \%$ neutral buffered formalin solution and stored at room temperature overnight. Samples were shipped to Pro Path (Dallas, TX, USA) the next day for processing by paraffin embedding. Tissue sections were processed for immunohistochemistry staining for procollagen 1, fibrillin 1 surrogate stain amyloid P, and mucins (dermal and epidermal; by both colloidal iron and Alcian blue staining methods). Expression of these markers was graded by an expert grader using the following scale (half-points were acceptable):

Procollagen 1: cytoplasmic stain in dermal fibroblastic cells, scale refers to $\%$ of cells with positive staining:

$$
\begin{aligned}
& 1=<5 \% \text { cells } \\
& 2=5 \%-10 \% \text { cells } \\
& 3=10 \%-20 \% \text { cells } \\
& 4=20 \%-30 \% \text { cells } \\
& 5=>30 \% \text { cells }
\end{aligned}
$$

Fibrillin 1 surrogate staining using amyloid P:

$0=$ within normal limits

$1=$ mild increase in reticulin fibers

2 =moderate increase in reticulin fibers

$3=$ marked, diffuse increase in dermal reticulin fibers

Mucin:

$0=$ normal

$1=$ mild increase in interstitial mucin

$2=$ moderate increase in interstitial mucin

3 =marked mucin deposition

\section{Immunohistochemistry}

\section{Procollagen I}

Sample slides were processed for immunohistochemistry staining for procollagen type 1 performed using MAB1912 anti-procollagen type I antibody, N-terminus, clone M-58 from EMD Millipore (Billerica, MA, USA), according to the manufacturer's directions. Antibody dilutions of 1:100 to $1: 1,000$ were used, after treatment with $1 \%$ trypsin, 20 minutes at room temperature.

\section{Fibrillin I}

Sample slides were processed for immunohistochemistry staining for fibrillin 1 surrogate stain amyloid $\mathrm{P}$ using antiserum amyloid P antibody [EP1018Y] (ab45151), a rabbit monoclonal antibody to serum amyloid $\mathrm{P}$, from Abcam (Cambridge, MA, USA) according to the manufacturer's directions. Antibody dilutions of 1:100 to 1:250 were used. Heat mediated antigen retrieval with citrate buffer $\mathrm{pH} 6$ was performed before commencing with immunohistochemistry staining protocol.

\section{Mucins}

Two different histology methods were employed for epidermal and dermal mucin staining, colloidal iron staining and Alcian blue staining before and after digestion with hyaluronidase; the latter method was used to confirm the results of the colloidal iron staining method after the removal of HA-related proteoglycans with hyaluronidase.

\section{Colloidal iron staining}

Skin biopsy samples were fixed in 10\% formalin for 20-24 hours prior to embedding in paraffin. Fixed tissue was cut at 4 microns onto positively charged slides and the sections deparaffinized and rehydrated in distilled water. Slides were rinsed briefly in $12 \%$ acetic acid solution and placed in working colloidal iron solution for 1 hour (Muller's colloidal iron solution + glacial acetic acid + deionized water). Slides were 
then immersed in ferrocyanide-hydrochloric acid solution for 20 minutes at room temperature. Following a rinse in tap water, the slides were counterstained with nuclear fast red solution for 5 minutes. Slides were then rinsed again in tap water, dehydrated in 95\% alcohol and absolute alcohol, cleared in xylene $(\times 2)$, and coverslipped. Slides were visualized via conventional light microscopy for mucin deposition within the epidermis and dermis (stringy-blue hue), and graded on the aforementioned semiquantitative scale.

\section{Alcian blue staining before and after digestion with hyaluronidase}

Skin biopsy samples were fixed in 10\% formalin for 20-24 hours prior to embedding in paraffin. Fixed tissue was cut at 4 microns onto positively charged slides and the sections deparaffinized and rehydrated in distilled water. Slides were placed in 3\% acetic acid solution for 3 minutes, and then in Alcian blue solution ( $\mathrm{pH} 2.5,1 \%$ Alcian blue 8GX, 3\% acetic acid, thymol) for 30 minutes. Following a tap water rinse, slides were incubated in $0.5 \%$ periodic acid for 10 minutes, rinsed, and then placed in Schiff's reagent for 10 minutes. Slides were then rinsed again in tap water, dehydrated in $95 \%$ alcohol and absolute alcohol, cleared in xylene $(\times 2)$, and coverslipped. Slides were visualized via conventional light microscopy for mucin deposition within the epidermis and dermis (stringy-blue hue), and graded on the aforementioned semiquantitative scale.

For hyaluronidase treatment, deparaffinized slides (4 microns) were treated with hyaluronidase solution $(0.05 \mathrm{~g}$ lyophilized hyaluronidase in $100 \mathrm{~mL}$ of $0.1 \mathrm{M}$ hyaluronidase buffer) for 1 hour at $37^{\circ} \mathrm{C}$. Following a tap water rinse, slides were stained per Alcian blue protocol and visualized/graded accordingly.

The difference between before digest and after digest was the final score for analysis (all postdigest scores were 0 ).

\section{Statistical considerations}

The per-protocol population was the primary population for all statistical analyses. The per-protocol population included all subjects who received treatment and completed the study in general accordance with the protocol. Only the data of completed subjects were analyzed.

A mixed effect model was built at each available postbaseline visit for biopsy grading scores. In the model, score is the response measurement, test site is the fixed effect, and subject is the random effect. Tukey's honestly significant difference test was used for pairwise site comparison to find which sites' means were different from one another.

All statistical tests were two-sided at significance level alpha $=0.05 . P$-values were reported to three decimal places $(0.000)$.
Statistical analyses were performed using SAS software version 9.30 series (SAS Statistical Institute, Cary, NC, USA).

\section{Results}

\section{Subject disposition and demographics}

Five subjects were enrolled and completed the study. Biopsy samples were obtained from the first four subjects who returned for the biopsy visit. Subject disposition and demographics are shown in Table 1. All five subjects enrolled were Caucasian females, median age of 49 years (median age 51 years for the four biopsy subjects). Four of the five subjects (three of the four biopsy subjects) had Fitzpatrick skin type II and one subject had Fitzpatrick skin type III.

\section{Biopsy analysis results}

Biopsy analyses results are presented in Tables 2 and 3.

Biopsy results for the presence of fibrillin/amyloid $\mathrm{P}$ indicate a statistically significant difference in favor of the test material (mean score of 1.25) and the reference control (mean score of 1.50), when compared to the negative control (mean score of 0.25).

Biopsy results for presence of epidermal mucins (colloidal iron and Alcian blue/hyaluronidase staining methods) indicate a statistically significant difference in favor of the test material (mean score of 1.25) and the reference control (mean score of 1.50 ), when compared to the negative control (mean score of 0.50 ).

Biopsy results for presence of procollagen I indicate a greater percentage of cells with positive staining for the test material (mean score of 1.25) and the reference control (mean score of 1.50), compared to the negative control (mean score of 1.00 ), though the differences were not statistically significant.

Biopsy results for presence of dermal mucin indicate a greater presence for the test material (mean score of 1.75) and the reference control (mean score of 1.25), compared to the negative control (mean score of 1.00), though the difference was not statistically significant.

Table I Subject disposition and demographics

\begin{tabular}{lll}
\hline & $\begin{array}{l}\text { All subjects } \\
(\mathbf{N}=\mathbf{5})\end{array}$ & $\begin{array}{l}\text { Biopsy subjects } \\
\mathbf{( N = 4 )}\end{array}$ \\
\hline $\begin{array}{l}\text { Age (years) } \\
\text { Mean (SD) }\end{array}$ & $50(3.9)$ & $5 \mathrm{I}(3.7)$ \\
$\begin{array}{l}\text { Median (min, max) } \\
\text { Sex, } \mathbf{n}(\%)\end{array}$ & $49(46,55)$ & $5 \mathrm{I}(47,55)$ \\
$\begin{array}{l}\text { Female } \\
\text { Ethnicity/race, n (\%) }\end{array}$ & $5(100)$ & $4(100)$ \\
$\begin{array}{l}\text { Caucasian } \\
\text { Fitzpatrick skin type, } \mathbf{n}(\%)\end{array}$ & $5(100)$ & $4(100)$ \\
II & $4(80)$ & $3(75)$ \\
III & $\mathrm{I}(20)$ & $\mathrm{I}(25)$ \\
\hline
\end{tabular}

Abbreviations: SD, standard deviation; min, minimum; max, maximum. 
Table 2 Descriptive statistics for biopsy results at day 12 ( $N=4$ subjects)

\begin{tabular}{|c|c|c|c|c|c|}
\hline \multicolumn{6}{|l|}{ Treatment } \\
\hline \multicolumn{6}{|l|}{ Procollagen I } \\
\hline Test material & 1.25 & 0.50 & 1.00 & 1.00 & 2.00 \\
\hline Reference control & 1.50 & 0.58 & 1.00 & 1.50 & 2.00 \\
\hline Negative control & 1.00 & 0.00 & 1.00 & 1.00 & 1.00 \\
\hline \multicolumn{6}{|l|}{ Fibrillin/amyloid $\mathbf{P}$} \\
\hline Test material & 1.25 & 0.50 & 1.00 & 1.00 & 2.00 \\
\hline Reference control & 1.50 & 0.58 & 1.00 & 1.50 & 2.00 \\
\hline Negative control & 0.25 & 0.50 & 0.00 & 0.00 & 1.00 \\
\hline \multicolumn{6}{|c|}{ Epidermal mucins (colloidal iron) } \\
\hline Test material & 1.25 & 0.50 & 1.00 & 1.00 & 2.00 \\
\hline Reference control & 1.50 & 0.58 & 1.00 & 1.50 & 2.00 \\
\hline Negative control & 0.50 & 0.58 & 0.00 & 0.50 & 1.00 \\
\hline \multicolumn{6}{|l|}{ Dermal mucin } \\
\hline Test material & 1.75 & 0.96 & 1.00 & 1.50 & 3.00 \\
\hline Reference control & 1.25 & 0.50 & 1.00 & 1.00 & 2.00 \\
\hline Negative control & 1.00 & 0.00 & 1.00 & 1.00 & 1.00 \\
\hline
\end{tabular}

Notes: Fibrillin, epidermal and dermal mucin are rated on four point (0-3) scale: $0=$ normal, $3=$ marked increased staining. Procollagen is rated on a 5 point $(I-5)$ scale: $I$ $=<5 \%$ cells positive, $5=>30 \%$ cells positive.

Table 3 Comparison details between test products for biopsies at day 12

\begin{tabular}{|c|c|c|c|c|c|}
\hline Parameter & $\begin{array}{l}\text { Overall } \\
P \text {-value } \\
\end{array}$ & Comparison & $\begin{array}{l}\text { Estimated mean } \\
\text { difference }\end{array}$ & $\begin{array}{l}\text { Standard } \\
\text { error }\end{array}$ & $\begin{array}{l}\text { Pairwise } \\
P \text {-value }\end{array}$ \\
\hline \multirow[t]{3}{*}{ Procollagen I } & 0.244 & Test material vs reference control & -0.25 & 0.26 & 0.632 \\
\hline & & Test material vs negative control & 0.25 & 0.26 & 0.632 \\
\hline & & Reference control vs negative control & 0.50 & 0.26 & 0.220 \\
\hline \multirow[t]{3}{*}{ Fibrillin/amyloid P } & 0.002 & Test material vs reference control & -0.25 & 0.20 & 0.483 \\
\hline & & Test material vs negative control & 1.00 & 0.20 & 0.006 \\
\hline & & Reference control vs negative control & 1.25 & 0.20 & 0.002 \\
\hline Epidermal mucins & 0.007 & Test material vs reference control & -0.25 & 0.20 & 0.483 \\
\hline \multirow[t]{2}{*}{ (Colloidal iron or Alcian blue/hyaluronidase) } & & Test material vs negative control & 0.75 & 0.20 & 0.024 \\
\hline & & Reference control vs negative control & 1.00 & 0.20 & 0.006 \\
\hline Dermal mucin & 0.178 & Test material vs reference control & 0.50 & 0.35 & 0.392 \\
\hline \multirow[t]{2}{*}{ (Colloidal iron or Alcian blue/hyaluronidase) } & & Test material vs negative control & 0.75 & 0.35 & 0.165 \\
\hline & & Reference control vs negative control & 0.25 & 0.35 & 0.768 \\
\hline
\end{tabular}

Notes: a ${ }^{P}$-value was calculated using a mixed effect model where test product is a fixed effect and subject is a random effect. Tukey's honestly significant difference test was used for pairwise treatment comparison. The null hypothesis is that the mean scores are equal between treatments.

A

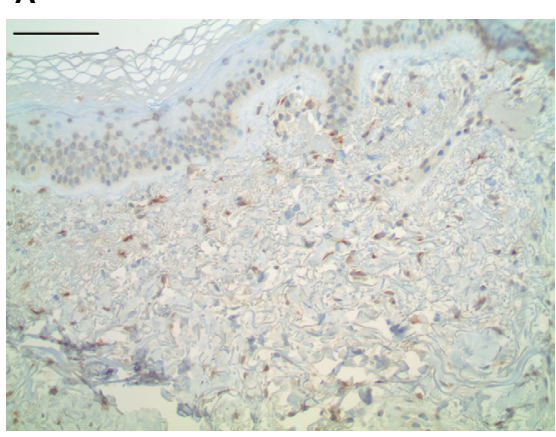

B

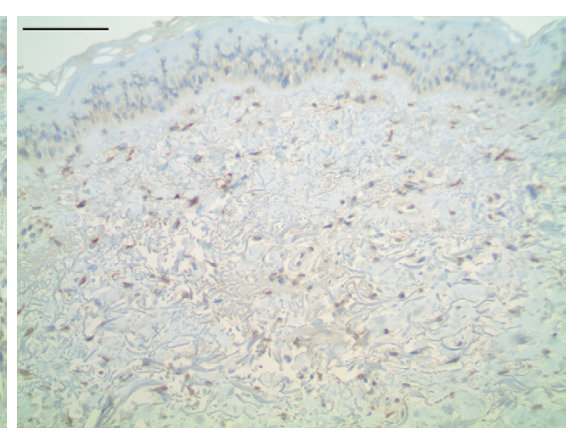

C

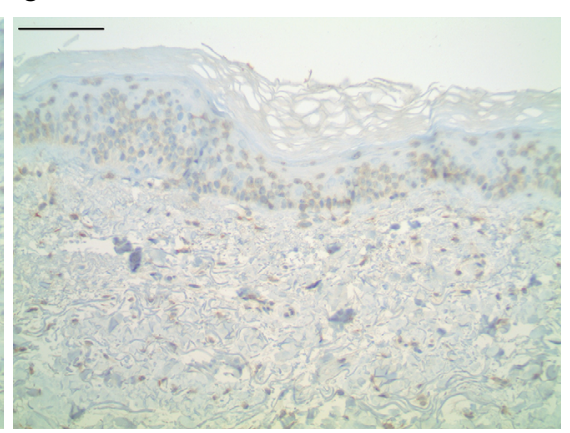

Figure I Procollagen I dermal histology.

Notes: Immunohistochemical staining for procollagen I, showing an increased level of staining in both the test material (A) (subject 4_site I_procollagen a) and reference control (B) (subject 4_site 2_procollagen b), when compared to negative control (C) (subject 4_site 3_procollagen c). Scale bar =200 $\mu$ m. 
A

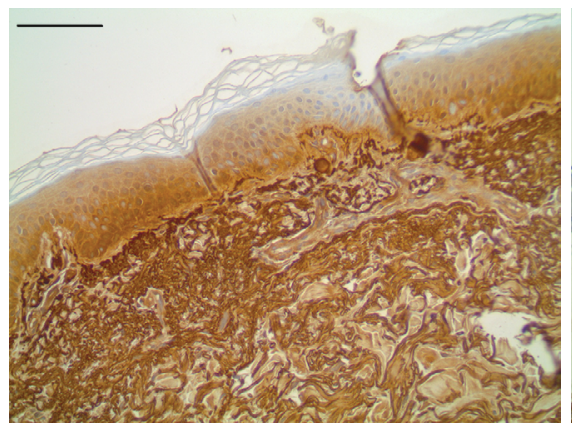

B

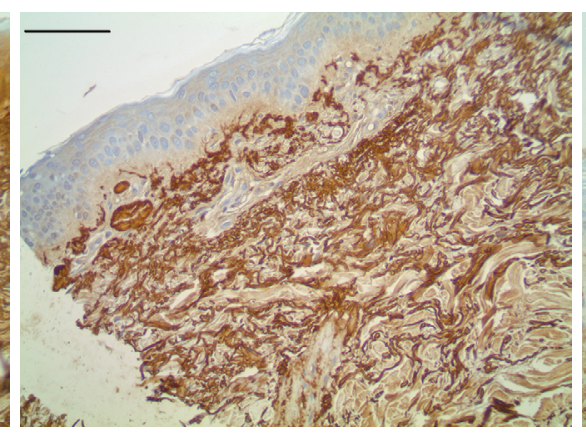

C

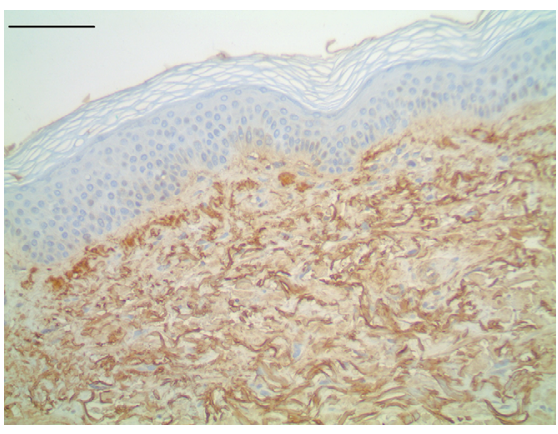

Figure 2 Fibrillin I dermal histology.

Notes: Immunohistochemical staining for amyloid P (fibrillin surrogate marker), showing increased fibrillin signal in both the test material (A) (subject 4_site I_fibrillin_amyp a), and reference control (B) (subject4_site2_fibrillin_amyp b), when compared to the negative control (C) (subject4_site3_fibrillin_amyp c). Scale bar =200 $\mu$ m.

A

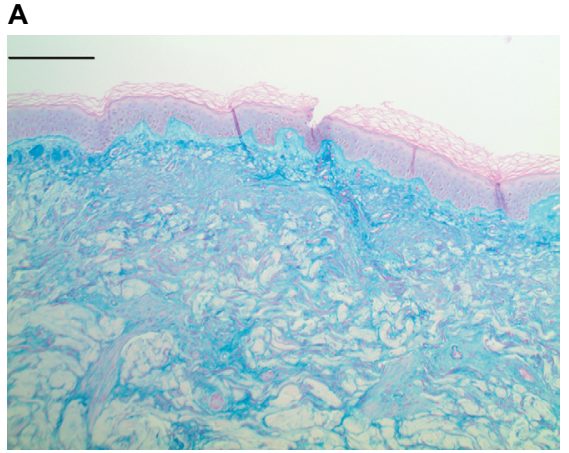

B

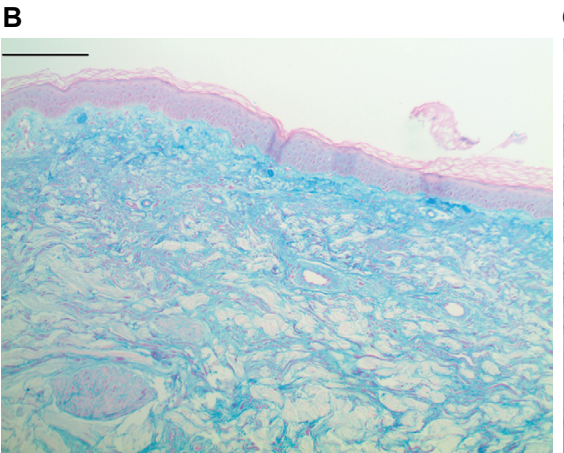

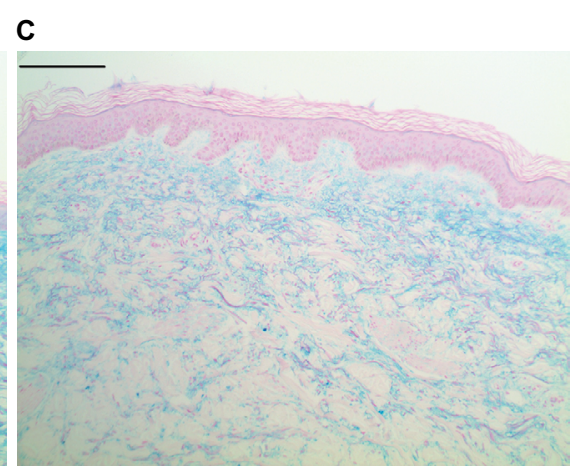

Figure 3 Epidermal and dermal mucin histology.

Notes: Histochemical staining for mucin via colloidal iron to highlight both epidermal and dermal mucins. An increased amount of mucin is seen in both compartments in both the test (A) (subject4_sitel_colfe_orig a) and reference material (B) (subject4_site2_colfe_orig b) compared to the negative control (C) (subject4_site3_colfe_orig c). Scale bar $=200 \mu \mathrm{m}$.

\section{Safety results}

No adverse events were observed or reported by subjects during the course of the study, including 10 days postbiopsy at the time of removal of biopsy stitches.

Under the exposure conditions of this test, the test material, reference control, and untreated negative control were all classified as "questionable/weak skin irritant," the lowest classification allowed by the test site protocol, and there was no statistically significant difference $(P>0.05)$ among the three sites.

\section{Discussion}

This study was conducted to extend the findings of in vitro and pilot clinical studies using sodium copper chlorophyllin complex and to further investigate the mechanism of action of the complex in the ECM.

A previous study demonstrated inhibition of hyaluronidase activity in vitro by sodium copper chlorophyllin complex. ${ }^{10}$ Pilot clinical studies demonstrated that a topical formulation containing a liposomal dispersion of sodium copper chlorophyllin complex was clinically effective and well tolerated for the treatment of mild-to-moderate photodamage and solar lentigines, ${ }^{11}$ mild-to-moderate acne and large visible pores, ${ }^{12}$ and demonstrated a reduction in facial redness, decreased pore size, and improved skin texture. ${ }^{13}$

In the present study, overall results from this single center, randomized, controlled, 12-day clinical trial indicate that both the test material Formula DR198-02 containing 0.05\% sodium copper chlorophyllin complex and the positive control, tretinoin cream $0.025 \%$, increased the presence of procollagen 1 , fibrillin 1, and epidermal and dermal mucins, which are biomarkers of dermal repair, compared to the negative control. The changes in fibrillin 1 and epidermal mucins for both the test material and the positive control were statistically significant $(P<0.05)$ when compared to no treatment. It was not possible to directly assess HA content in the biopsy specimens; therefore, the results reflect the effects of the test material on GAGs and fibrous proteins in general and are not specific to HA. However, assessments of changes in epidermal and dermal mucins were used as a surrogate method for measuring changes in hyaluronan in this study. Since hyaluronidase digestion in the Alcian blue staining method removed all increases observed for mucins, this would suggest that the changes observed before digestion were from HA, and not other GAGs or protein-bound 
mucopolysaccharides. The increases in epidermal and dermal mucins demonstrated by the $0.05 \%$ sodium copper chlorophyllin gives in vivo support to the hyaluronidase inhibitory activity of this compound that has previously demonstrated strong in vitro anti-hyaluronidase activity. ${ }^{10}$

Analyses of the biopsy samples showed a statistically significant difference in favor of the test material and reference control for the presence of fibrillin/amyloid $\mathrm{P}$ and presence of epidermal mucins (Col/FE) when compared to the negative control (untreated). There was no statistical difference between the test material and reference control for any of the increases in the measured biomarkers.

Tretinoin (retinoic acid) was used as the positive control in this study. Retinoic acid is a widely used treatment and considered the gold standard for photoaged skin. ${ }^{14,15}$ It has been demonstrated that all-trans-retinoic acid increases the synthesis of hyaluronan and upregulates hyaluronan synthase 2 (Has2) and hyaluronan synthase 3 (Has3) gene expression in cultured rat epidermal keratinocytes, and appears to be mediated by epidermal growth factor. ${ }^{16,17}$ A recent publication describes a topically applied antiaging product that improved the appearance of fine lines/wrinkles and skin hydration, presumably by promoting restoration of endogenous epidermal HA homeostasis. ${ }^{18}$

Two products, Rejuvaphyl Rejuvenating Complex LS and Rejuvaphyl Rejuvenating Complex HS from MDRejuvena, contain both sodium copper chlorophyllin complex and retinol, thereby providing the potential for a dual approach to reversing age-related decreases in $\mathrm{HA}$ in the skin: inhibition of the breakdown of HA by sodium copper chlorophyllin complex by inhibition of hyaluronidase, and stimulation of HA synthases by retinol.

\section{Conclusion}

This clinical study demonstrates a statistically significant improvement in repair of photoaged skin by topical treatment with a test formulation containing a liposomal dispersion of $0.05 \%$ sodium copper chlorophyllin, based on changes in key epidermal and dermal biomarkers. The degree of repair seen with the sodium copper chlorophyllin complex and the positive control (tretinoin cream, United States Pharmacopeia $0.025 \%$ ), based on immunohistological analysis, was not statistically different. The results support and validate recently published in vitro antihyaluronidase activity for sodium copper chlorophyllin complex. Topical retinoid and sodium copper chlorophyllin complex for repair of photoaged skin should be evaluated further in this biopsy model to quantify whether there is an additive or synergistic response with this unique combination of topical agents.

\section{Acknowledgments}

Medical writing assistance was provided by Georgia Theofan, PhD of ProPharmaCon LLC, San Diego, CA, USA. MDRejuvena, Inc. (San Diego, CA) sponsored the clinical study described in this paper.

\section{Disclosure}

The first author, John P McCook, is Managing Partner of CHL Industries, LLC, the developer and licensor of topical sodium copper chlorophyllin complex technology described in this paper. The authors report no other conflicts of interest in this work.

\section{References}

1. Manuskiatti W, Maibach HI. Hyaluronic acid and skin: wound healing and aging. Int J Dermatol. 1996;35(8):539-543.

2. Meyer LJ, Stern R. Age-dependent changes of hyaluronan in human skin. J Invest Dermatol. 1994;102(3):385-389.

3. Stern R, Maibach HI. Hyaluronan in skin: aspects of aging and its pharmacologic modulation. Clin Dermatol. 2008;26((2):106-122.

4. Mio K, Stern R. Inhibitors of the hyaluronidases. Matrix Biol. 2002;21(1):31-37.

5. Girish KS, Kemparaju K, Nagaraju S, Vishwanath BS. Hyaluronidase inhibitors: A biological and therapeutic perspective. Curr Med Chem. 2009;16(18):2261-2288.

6. Tumolo T, Lanfer-Marquez UM. Copper chlorophyllin: a food colorant with bioactive properties? Food Res Int. 2012;46(2):451-459.

7. Bowers WF. Chlorophyll in wound healing and suppurative disease. $\mathrm{Am}$ J Surg. 1947;73((1):37-50.

8. Carpenter EB. Clinical experience with chlorophyll preparations with particular reference to chronic osteomyelitis and chronic ulcers. Am J Surg. 1949;77(2):167-171.

9. Smith RG. Enzymatic debriding agents: an evaluation of the medical literature. Ostomy Wound Manage. 2008;54(8):16-34.

10. McCook JP, Dorogi PL, Vasily DB, Cefalo DR. In vitro inhibition of hyaluronidase by sodium copper chlorophyllin complex and chlorophyllin analogs. Clin Cosmet Investig Dermatol. 2015;8:443-448.

11. Sigler ML, Stephens TJ. Assessment of the safety and efficacy of topical copper chlorophyllin in women with photodamaged facial skin. $J$ Drugs Dermatol. 2015;14(4):401-404.

12. Stephens TJ, McCook JP, Herndon JH. Pilot study of topical copper chlorophyllin complex in subjects with facial acne and large pores. $J$ Drugs Dermatol. 2015;14(6):589-592.

13. Vasily DB. Topical treatment with liposomal sodium copper chlorophyllin complex in subjects with facial redness and erythematotelangiectatic rosacea: case studies. J Drugs Dermatol. 2015;14(10):1157-1159.

14. Watson RE, Long SP, Bowden JJ, Bastrilles JY, Barton SP, Griffiths CE. Repair of photoaged dermal matrix by topical application of a cosmetic 'antiageing' product. Br J Dermatol. 2008;158(3):472-477.

15. Singh M, Griffiths CE. The use of retinoids in the treatment of photoaging. Dermatol Ther. 2006;19(5):297-305.

16. Pasonen-Seppanen SM, Maytin EV, Torronen KJ, et al. All-trans retinoic acid-induced hyaluronan production and hyperplasia are partly mediated by EGFR signaling in epidermal keratinocytes. J Invest Dermatol. 2008;128((4):797-807.

17. Saavalainen K, Pasonen-Seppanen S, Dunlop TW, Tammi R, Tammi MI, Carlberg C. The human hyaluronan synthase 2 gene is a primary retinoic acid and epidermal growth factor responding gene. J Biol Chem. 2005;280(15):14636-14644.

18. Narurkar VA, Fabi SG, Bucay VW, et al. Rejuvenating hydrator: restoring epidermal hyaluronic acid homeostasis with instant benefits. $J$ Drugs Dermatol. 2016;15((1 Suppl 2):s24-s37. 


\section{Publish your work in this journal}

Clinical, Cosmetic and Investigational Dermatology is an international, peer-reviewed, open access, online journal that focuses on the latest clinical and experimental research in all aspects of skin disease and cosmetic interventions. This journal is included on PubMed. The manuscript management system is completely online

Submit your manuscript here: https://www.dovepress.com/clinical-cosmetic-and-investigational-dermatology-journal

and includes a very quick and fair peer-review system, which is all easy to use. Visit http://www.dovepress.com/testimonials.php to read real quotes from published authors 